\title{
Quantifying the Performance of Protein-Resisting Surfaces at Ultra-Low Protein Coverages using Kinesin Motor Proteins as Probes**
}

\author{
By Parag Katira, Ashutosh Agarwal, Thorsten Fischer, Hsien-Yeh Chen, Xuwei Jiang, Joerg Lahann, \\ and Henry Hess*
}

Surfaces resistant to protein adsorption are very desirable for a variety of applications in biomedical engineering and bionanotechnology, since protein adsorption is often the first step in a cascade of events leading to systems failure. Initial efforts to create adsorption-resistant surfaces succeeded in reducing the adsorption by $80 \%$ compared to untreated surfaces to $100 \mathrm{ng} \mathrm{cm}^{-2}$ by employing poly(ethylene glycol) coatings. ${ }^{[1]}$ Recently, optimization of brush density and morphology has reduced adsorption to $1 \mathrm{ng} \mathrm{cm}^{-2}$ or less. ${ }^{[2]}$ These coverages, equal to about one tenth of a percent of a monolayer, represent the detection limit for several characterization techniques, including SPR ${ }^{[2,3]}$ and radiolabeling. ${ }^{[4]}$ However, biological effects can be observed for protein coverages below this detection limit. For example, adsorption of blood proteins can initiate the intrinsic coagulation cascade at low coverage. ${ }^{[5]}$ Therefore more sensitive techniques for the measurement of protein adsorption are needed. Moreover, if protein adsorption is exceedingly slow, higher sensitivity would permit an accelerated quantification of the performance of the surface (e.g. within hours instead of days).

Non-fouling surfaces are also a critical part of hybrid nanodevices, which utilize precisely positioned biomolecules in an artificial environment. ${ }^{[6]}$ For example, controlled adsorption of kinesin, ${ }^{[7-14]}$ myosin, ${ }^{[15-17]}$ and F1-ATPase motors ${ }^{[18]}$ has been utilized for the design of molecular shuttles and nanopropellers. Adsorption of motor proteins outside the intended regions at densities down to one motor per square micrometer

[*] Prof. H. Hess, P. Katira, A. Agarwal, Dr. T. Fischer Department of Materials Science and Engineering University of Florida, Gainesville, FL 32611-6400 (USA)

E-mail: hhess@mse.ufl.edu

H. Y. Chen, Dr. X. Jiang, Prof. J. Lahann

Department of Chemical Engineering, Department of Materials Engineering, and Macromolecular Science and Engineering Program University of Michigan, Ann Arbor, MI 48109 (USA)

[*** P.K. and A.A. contributed equally to this work. H.H. gratefully acknowledges financial support from the DOE Office of Basic Energy Sciences, the DARPA-DSO Biomolecular Motors Program (FA955005-1-366). T.F. thanks the Alexander-von-Humboldt foundation for support through a Feodor Lynen fellowship. J.L. gratefully acknowledges support from the NSF in form of a CAREER grant (DMR0449462) and funding form the NSF under the MRI program (DMR 0420785).
( $\left.0.04 \mathrm{ng} \mathrm{cm}^{-2}\right)$ can lead to loss of device function, since individual motors can already bind and transport the associated filaments outside their intended tracks.

The binding of associated filaments, such as microtubules or actin filaments, is readily observed by fluorescence microscopy, since these filaments are composed of thousands of protein subunits and carry typically at least a thousand covalently linked fluorophores. ${ }^{[19,20]}$ Howard et al. demonstrated in 1989 that observing the attachment of microtubules from solution to surface-adhered kinesin motors enables the determination of motor densities as low as 2 proteins per $\mu \mathrm{m}^{2}$ by measuring the rate of microtubule attachment. ${ }^{[21,22]}$ Attachment rate measurements have subsequently been adapted to the determination of relative kinesin motor activity on different surfaces $^{[9]}$ and to the evaluation of guiding structures for microtubule transport ${ }^{[10,23]}$ Since kinesins long tail domain evolved to efficiently connect to cargo, we hypothesize that it can serve as a particularly efficient probe for attachment points on the surface. ${ }^{[2,25]}$. Landing rate measurements enable the measurement of absolute coverages of functional kinesins in the range of $0.004-1 \mathrm{ng} \mathrm{cm}^{-2}$, thus enabling to differentiate the performance of even the best non-fouling surfaces. While landing rate measurements in effect count individual proteins, their complexity is low compared to single molecule fluorescence measurements, ${ }^{[26,27]}$ due to the availability of a kinesin/ microtubule kit (Cytoskeleton Inc.) and the high brightness of fluorescent microtubules which can be imaged with a standard fluorescence microscope.

In the following, we demonstrate that the quantification of ultra-low kinesin coverages by landing rate measurements is a valuable tool in determining the performance of novel and established coatings with outstanding resistance to protein adsorption (see Scheme 1 for an example of a designer surface). Specifically, we will describe the adsorption model underlying the method, present experiments which demonstrate the determination of kinesin coverages on fouling and nonfouling surfaces, and discuss the advantages and limitations of the proposed method.

Adsorption Model. Microtubule attachment rate measurements are interpreted in the context of a two stage adsorption model: First, kinesin molecules adsorb from solution to the surface, filling a fraction of the available binding sites. Second, the kinesin solution is replaced by a microtubule solution and microtubules adsorb specifically to the kinesin motors bound 


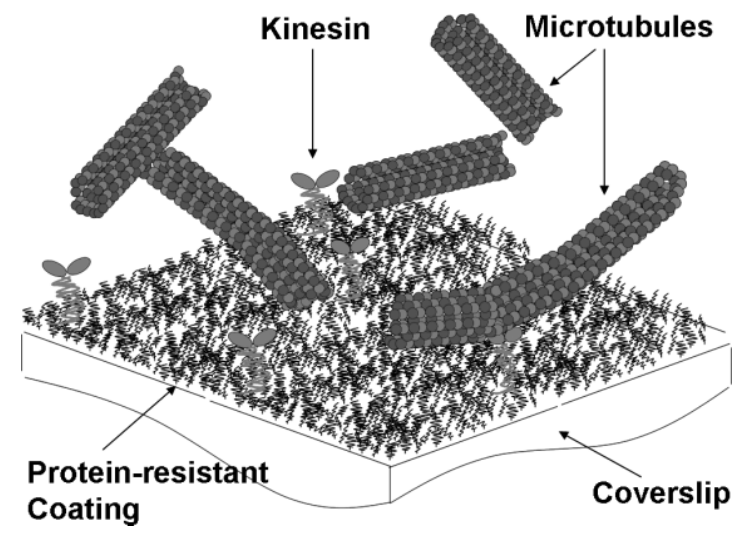

Figure 1. Sketch of principle: Adsorption of kinesin motor proteins to a non-fouling surface can be quantified by measuring initial microtubule landing rates. In the first step, solution-based kinesin scans the surface for 'defect sites' and adsorbs to a fraction of or all available sites. In the second step, the unbound motors in the solution are replaced by a microtubule solution containing AMP-PNP and the landing rate of microtubules on the surface is measured. This along with measurement of the average length of landed microtubules and measurement of the diffusion limited maximum landing rate on a fouling surface provides an estimate of the kinesin surface density.

to the surface (Fig. 1). Both processes, kinesin adsorption and microtubule binding, are assumed to be irreversible on the timescale of the experiment $(<1 \mathrm{hr})$, since the solution exchange removes weakly bound motors, and the use of AMP-PNP (a nonhydrolyzable ATP analogue ${ }^{[28]}$ ) prevents detachment of microtubules from motors.

The surface concentration of adsorbed kinesin motors obtained after a defined period (e.g. $5 \mathrm{~min}$ ) is then quantified

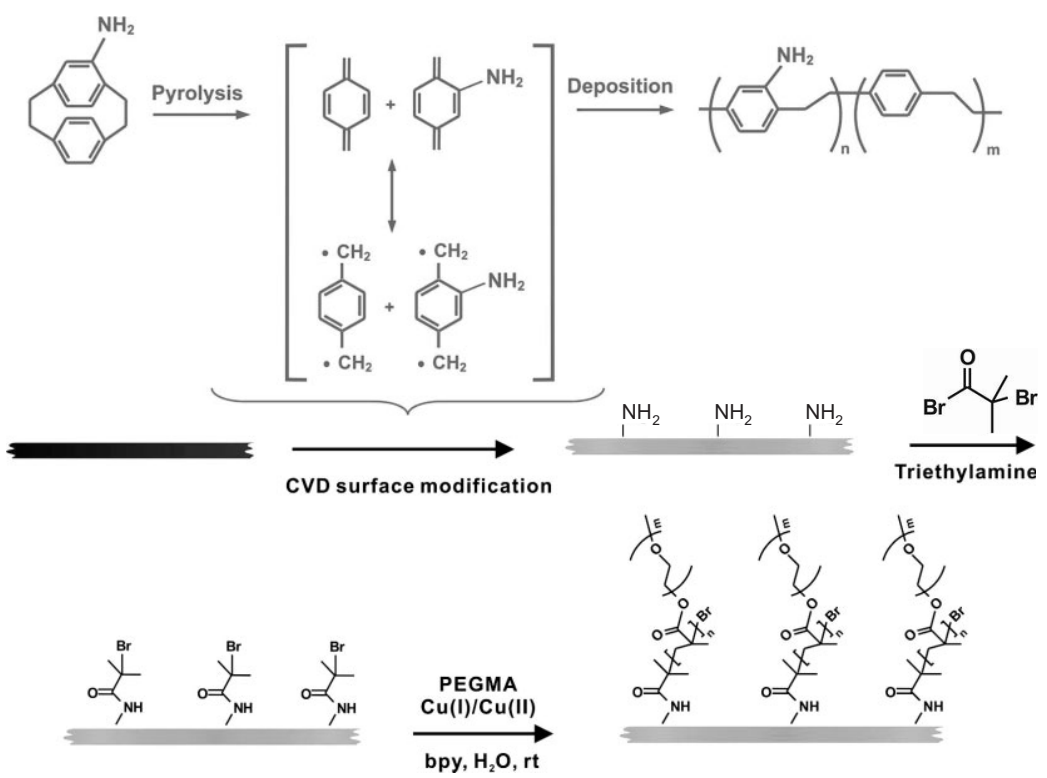

Scheme 1. Schematic description of the process used to prepare PEGMA-modified glass slides consisting of (i) CVD polymerization, (ii) coupling of the initiator, and (iii) ATRP coating of PEGMA. by measuring the initial landing rate $\mathrm{R}$ of microtubules on the coated surfaces, and the maximal, diffusion-limited landing rate $Z$ on a control surface densely coated with motor proteins. The initial landing rate is related to the motor density $\rho$ according to $R(\rho)=Z[1-\exp (-\rho A)]$, since the microtubules are present in excess in the solution. Here, $A=L w$ is the footprint of the microtubule, where the average length $\mathrm{L}$ is measured and the width is assumed to be $w=25 \mathrm{~nm}$. ${ }^{\text {[2] }}$

The initial landing rate $R$ begins to substantially deviate from the diffusion-limited maximal landing rate if $\rho A<1$, where $A \sim 0.1 \mu \mathrm{m}^{2}$ for typical microtubule lengths. Since the minimal measurable landing rate is on the order of $1 \mathrm{~mm}^{-2} \mathrm{~s}^{-1}$, and the diffusion limited landing rate $Z$ is on the order of $100 \mathrm{~mm}^{-2} \mathrm{~s}^{-1}$, microtubule landing rate measurements are sensitive to motor densities between $0.1 \mu \mathrm{m}^{-2}$ and $30 \mu \mathrm{m}^{-2}$.

It is well-established that direct adsorption of kinesin to fouling (glass) and moderately non-fouling (Pluronic F108 coating) surfaces leads to denaturation and loss of its microtubule-binding ability. ${ }^{[21,30]}$ However, if the surface is covered by either denatured kinesin motors or a blocking protein (e.g. albumin or casein) interstitial binding of kinesin tails to the surface results in high motor functionality. These observations are reproduced in our measurements (Fig. 2h), which show that direct absorption of kinesin to glass results in a non-linear increase of the landing rate as the amount of available kinesin is increased. In contrast, pre-coating of the glass, PU and Pluronic surfaces with casein results in an initially linear dependence of microtubule landing rate (Fig. 3 top) and surface density of active motors (Fig. 3 bottom) on the amount of available kinesin.

On these surfaces, the diffusion-limited landing rate is reached for moderate (20-fold) dilutions of the kinesin stock solution (Fig. 2 and 3), whose concentration can be calculated as $175 \mathrm{nM}$ (see Experimental). This value was used to determine dosage values in Figure 3. In all subsequent experiments, the diffusion-limited landing rate $Z$ for a given microtubule preparation is assumed to be equal to the observed landing rate on a casein-coated glass surfaces exposed to a $17.5 \mathrm{nM}$ kinesin solution (10-fold dilution of stock) for $5 \mathrm{~min}$.

Since physisorbed Pluronic F-108 reduces protein adsorption by $\sim 80 \%,{ }^{[31]}$ we interpret the observed four-fold lower density of microtubule-binding motors as a reflection of the reduced adsorption of casein. Kinesin contact to the bare surface will always lead to denaturation, whereas kinesin contact to adsorbed casein will result in a functional motor. Similarly, the density of active motors is $40 \%$ lower on casein-coated polyurethane (PU) surfaces compared to casein-coated glass surfaces.

With the principle of the kinesin/microtubule based assay established, we shifted our focus to two types of highly non-fouling surfaces, $(\mathrm{EG})_{3} \mathrm{OH}$-terminated SAMs and polyethylene 
glycol methacrylate (PEGMA) coated surfaces. To prepare protein-resistant PEGMA coatings (Scheme 1), we first introduced functional groups onto the substrates using chemical vapor deposition (CVD) polymerization of 4-amino [2.2]paracyclophane. CVD polymerization is a vapor-based coating approach that can be used to modify a wide range of different substrate materials and geometries with a series of different chemical groups. ${ }^{[32-34]}$ In this case, $50 \mathrm{~nm}$ thick films of poly(4-amino-p-xylylene-co-p-xylylene) were CVD deposited on glass substrates to provide free amino groups for further surface modification. ${ }^{[35]}$ The amino-functionalized coating was then reacted with 2-bromoisobutyryl bromide. In the final step, bromoisopropyl groups were used as initiators for surface-initiated atom transfer radical polymerization (ATRP) of poly(ethylene glycol) methyl ether methacrylate (PEGMA).

Direct adsorption of kinesin to these highly non-fouling surfaces led to low landing rates which did not significantly increase with increasing kinesin concentration in solution (Fig. 3). Precoating (EG) ${ }_{3} \mathrm{OH}$-terminated SAM surfaces with casein followed by exposure to $17.5 \mathrm{nM}$ of kinesin for 5 min reduced the observed microtubule landing rate from $\sim 31 \mathrm{~mm}^{-2} \mathrm{~s}^{-1}$ to $\sim 2 \mathrm{~mm}^{-2} \mathrm{~s}^{-1}$, corresponding to a 15 -fold reduction in kinesin surface density (data not shown).
Our interpretation of these observations is that the highly non-fouling surface helps conserve kinesin motor function after adsorption, and that casein acts as a competitor for a very limited number of adsorption sites. Furthermore, since the surface density of adsorbed kinesin does not linearly increase with kinesin dosage, the residual kinesin adsorption is not a consequence of kinesins slowly penetrating the coating. ${ }^{[36]}$ Instead, the surface density of adsorbed kinesins is equal to the density of defects in the coating.

$(\mathrm{EG})_{3} \mathrm{OH}$-terminated SAM surfaces, a widely studied model system, ${ }^{[37-39]}$ adsorbed an average of $4.2 \pm 0.7 \mu \mathrm{m}^{-2}$ $\left(0.17 \pm 0.03 \mathrm{ng} \mathrm{cm}^{-2}\right)$. This average is derived from three sets of identical surfaces prepared on different days (SAM1, SAM2 and SAM3). In comparison, the reported values measured using SPR for the adsorption of fibrinogen at hundred- to thousand-fold higher dosages are $0.35 \pm 1.75 \mathrm{ng} \mathrm{cm}^{-2}$ $\left(1 \mathrm{mg} \mathrm{mL}^{-1} \text { adsorbed for } 3 \mathrm{~min}\right)^{[40]}$ and $2.8 \pm 1.05 \mathrm{ng} \mathrm{cm}^{-2}$ $\left(1 \mathrm{mg} \mathrm{mL}^{-1}\right.$ adsorbed for $\left.30 \mathrm{~min}\right){ }^{[41]}$

PEGMA surfaces had exceptionally low landing rates as can be seen in Figure 2d-f. The average motor density on these surfaces is $0.16 \pm 0.02 \mu \mathrm{m}^{-2}\left(0.0064 \pm 0.0008 \mathrm{ng} \mathrm{cm}^{-2}\right.$; average of PEGMA1 and PEGMA2). These data suggest that PEGMA surfaces represent a significant improvement over $(\mathrm{EG})_{3} \mathrm{OH}$-terminated SAM surfaces.
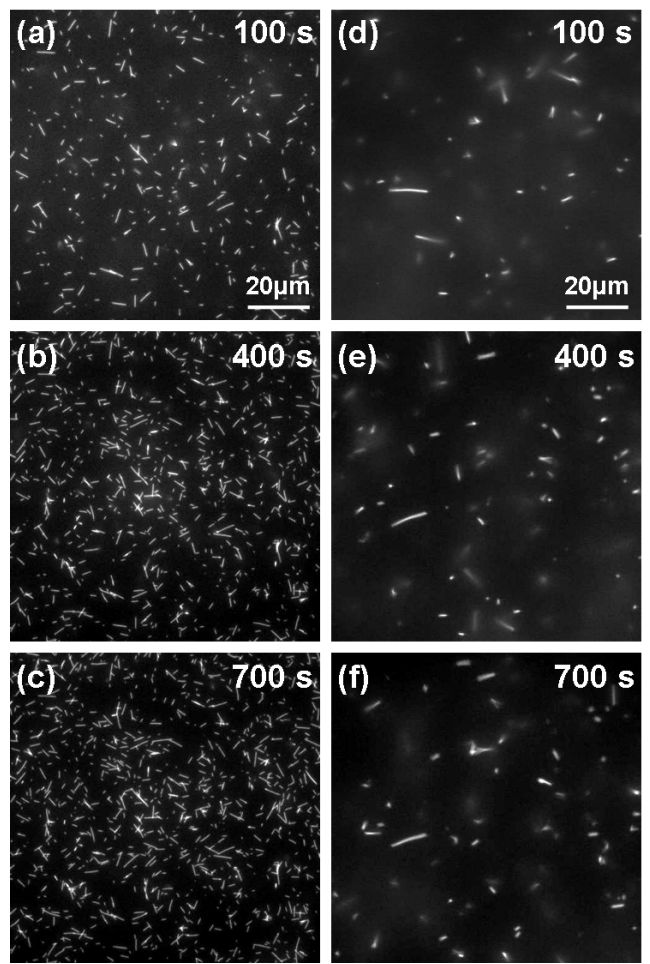
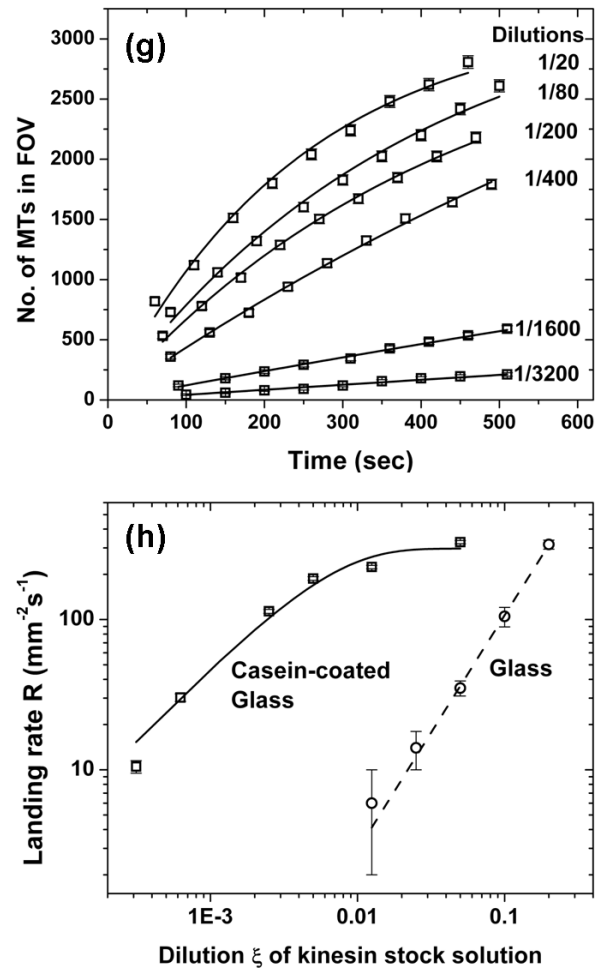

Figure 2. Measurement of kinesin surface densities from landing rates of microtubules. (a-c) Microtubules binding 100 , 400 , and $700 \mathrm{~s}$ after microtubule injection to a casein-coated glass surface exposed to kinesin solution twenty-fold diluted from stock ( $\sim 9 \mathrm{nM})$ for 5 min (d-f) Microtubules binding 100, 400, and $700 \mathrm{~s}$ after microtubule injection to a PEGMA surface exposed to kinesin solution twenty-fold diluted from stock ( 9 nM) for 5 min. The average length of microtubules for $(a-c)$ is $1.71 \pm 0.08 \mu \mathrm{m}$ and for $(d-f)$ is $2.29 \pm 0.11 \mu \mathrm{m}$. The field of view (FOV) was $200 \mu \mathrm{m} \times 200 \mu \mathrm{m}$ but (a-f) are cropped for clarity. (g) The number of microtubules attached to the surface as function of time for the casein-coated glass exposed to kinesin solutions diluted from the stock solution. (h) Landing rates R computed from the data shown in (g) and plotted against the concentration of the kinesin solution for casein-coated glass surfaces (open squares) and bare glass surfaces (open circles). 

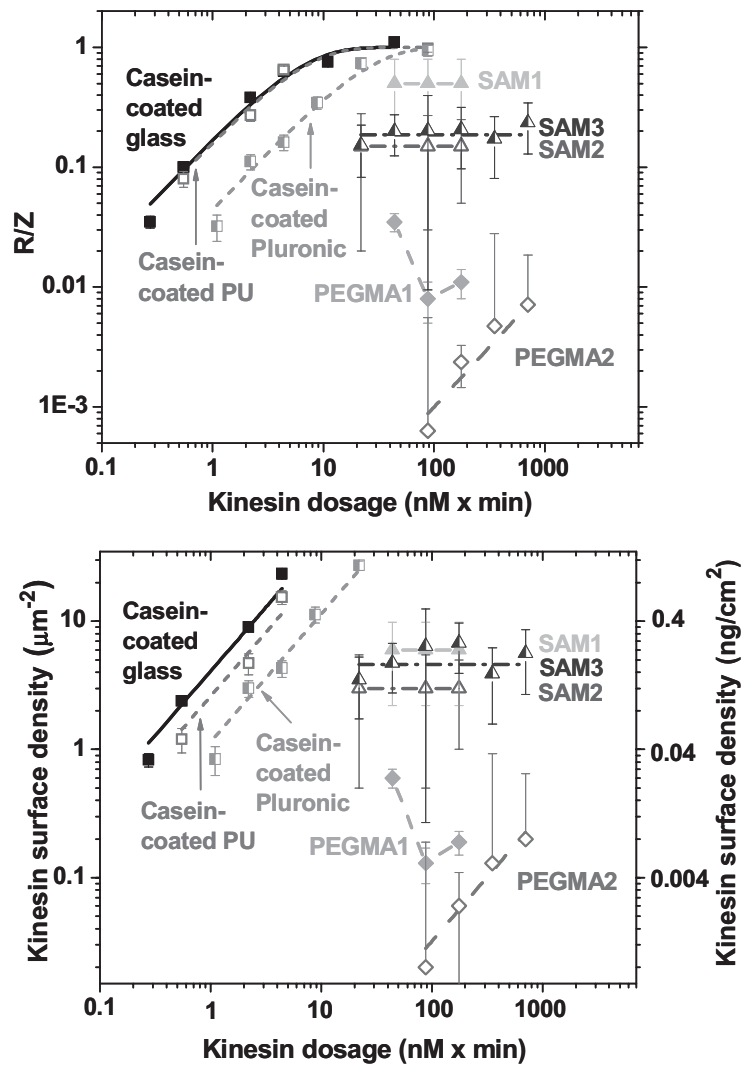

Figure 3. Top - The ratio of measured landing rate $\mathrm{R}$ to the diffusion-limited landing rate $Z$ as a function of the kinesin dosage for different surfaces. Bottom - The kinesin surface density as a function of kinesin dosage calculated from the ratio $R / Z$ and the average microtubule length.

In conclusion, kinesin protein adsorption followed by microtubule landing rate measurements enables the determination of active protein coverages between 0.1 and $30 \mu \mathrm{m}^{-2}$ $\left(0.004-1 \mathrm{ng} \mathrm{cm}^{-2}\right)$. This detection range extends the lower end of the detection range of established methods. In essence, microtubule landing rate measurements afford single molecule sensitivity by exploiting the thousand-fold amplification of a fluorescence signal provided by labeled microtubules. The detection limit can be further reduced by increasing the observable number of microtubule landing events (increasing the field of view, observation time, or microtubule solution concentration). It is possible, that the ability of kinesin to bind microtubules is reduced after adsorption to the highly nonfouling surfaces. However, this reduced activity affects primarily the absolute protein coverage calculated from the data, and not the relative performance of two highly non-fouling surfaces.

While the method does not determine the performance of non-fouling surfaces in blood serum or solutions of blood proteins, the reduced detection limit enables the quantification of adsorption events which would be invisible to established techniques. As a result, the performance of highly non-fouling surfaces can be determined and optimized. For example, the adsorption of kinesin to PEGMA surfaces is twenty-fold reduced compared to $(\mathrm{EG})_{3} \mathrm{OH}$-terminated SAM surfaces. We hope that the low technical requirements (fluorescence microscope with camera) and the commercial availability of a kinesin motility kit (Cytoskeleton Inc.) make this method widely accessible.

In the context of hybrid devices integrating motor proteins, the measurements demonstrate that the newly developed coatings can achieve the extreme degree of adsorption resistance desirable for the reliable placement of kinesin motors. Similar to such hybrid devices, biosensors utilizing nanowires or other nanostructures as transducing elements require highly adsorption resistant surfaces to maintain their performance advantages on the system level. These are only two examples of the diverse applications of high performance protein-resistant coatings in bionanotechnology.

\section{Experimental}

Preparation of Surfaces: For glass surfaces, coverslips (Fisherfinest, Premium Cover Glass, no 1, Fisher Scientific, Pittsburg, PA) were cleaned with ethanol and dried. For polyurethane (PU) surfaces, UV curable PU precursor NOA 73 (Norland Products Cranbury, NJ) was spincoated onto glass coverslips at $3500 \mathrm{rpm}$ for $40 \mathrm{~s}$ and cured for at least 2 hrs using a $365 \mathrm{~nm}$ UV lamp (Spectroline EN-280L, Spectronics, Westbury, NY).

Physisorbed pluronic surfaces were prepared according to [42]. Glass surfaces were cleaned twice by batch sonication for $15 \mathrm{~min}$ in $5 \%$ (v/v) Contrad 70 soap (Fisher Scientific, Pittsburg, PA). Prior to the second batch sonication, all samples were sonicated in deionized distilled water for $15 \mathrm{~min}$. After a final rinse with water and drying at $200{ }^{\circ} \mathrm{C}$ for $15 \mathrm{~min}$, the coverslips were treated with $5 \%$ dimethyldichlorosilane in toluene (v/v, Cylon CT, Supelco Inc., Bellefonte, PA) and rinsed twice with toluene and thrice with methanol. These visibly hydrophobic surfaces (contact angle $=86^{\circ}$ measured using a RameHart goniometer, Model 100-00, Rame-Hart, Mountain Lakes, NJ) were treated overnight with $2 \mathrm{mg} \mathrm{mL}^{-1}$ Pluronic F108 (BASF, Mount Olive, NJ) solution in water. Unbound Pluronic was removed from the surface by rinsing twice with water and once with BRB80 buffer (80 mM PIPES, $1 \mathrm{mM} \mathrm{MgCl}, 1 \mathrm{mM}$ EGTA, $\mathrm{pH}$ 6.9). After the Pluronic treatment, the contact angle changed to $71^{\circ}$ which matches the reported contact angles for physisorbed Pluronic [43].

Self-assembled monolayers terminated in $(\mathrm{EG})_{3} \mathrm{OH}$ were prepared by sputter-coating cleaned glass coverslips with a transparent gold layer (25 Å chromium and 150 Å gold) using a CMS-18 Multi Target Sputter Deposition machine (Kurt Lesker, Clairton, PA) [44], and immersing them overnight in $1 \mathrm{mM}$ solution of 1-mercapto-11-undecyl tri(ethylene glycol) (Asemblon Inc., Redmond, WA) prepared in 99.9\% methanol (Sigma-Aldrich, Saint Louis, MO) according to [41].

PEGMA Coatings: Unless otherwise specified, all chemicals were purchased from Aldrich. 2-bromoisobutyryl bromide (98\%), triethylamine (TEA, 99.5\%), $\mathrm{Cu}(\mathrm{I}) \mathrm{Br}$ (99.999\%), $\mathrm{Cu}(\mathrm{II}) \mathrm{Br}_{2}$ (Fluka, 99\%) were used as received. Hexanes were distilled over calcium hydride. 2,2'-dipyridyl (bpy, 99\%) was sublimed. Poly(ethylene glycol) methyl ether methacrylate (PEGMA, average $M_{\mathrm{n}} \sim 475$ ) was passed through a $20 \mathrm{~cm}$ column of inhibitor remover and stored at $-20^{\circ} \mathrm{C}$.

Glass slides were initially modified via CVD polymerization of 4-amino [2.2]paracyclophane using a custom-built installation [45]. $50 \mathrm{mg}$ of the paracyclophane were sublimed at $90^{\circ} \mathrm{C}$ and 0.12 Torr, thermally activated, and transferred into a deposition chamber, where the polymer film was deposited at $15^{\circ} \mathrm{C}$. Films made under these conditions had a thickness of $50-70 \mathrm{~nm}$ as determined by ellipsometry (EP3, Nanofilm AG). The resulting amino-functionalized glass slides were immersed into an anhydrous hexane solution containing $200 \mu \mathrm{L}$ 2-bromoisobutyryl bromide and $300 \mu \mathrm{L}$ TEA were added subse- 
quently. After incubation for three minutes at room temperature, the surface-modified glass slides were removed from the solution, washed sequentially with water and ethanol, and dried under a stream of nitrogen. Next, PEGMA $(40 \mathrm{~mL})$, deinoized water $(20 \mathrm{~mL})$, bpy (304 mg), and $\mathrm{Cu}(\mathrm{II}) \mathrm{Br}_{2}(40 \mathrm{mg})$ were charged into a Schlenk flask, stirred until homogeneous at room temperature, and degassed using three freeze-pump-thaw cycles. $\mathrm{CuBr}(86 \mathrm{mg})$ was then added under nitrogen purge with the contents in the flask being frozen. The flask was then evacuated and backfilled with nitrogen five times, and finally backfilled with nitrogen and warmed up to room temperature. The mixture in the flask was stirred until the formation of a homogeneous dark brown solution was observed. Finally, surface-modified glass slides were incubated with this solution in a nitrogen purged glove bag at room temperature for three hours. The substrates were then removed from PEGMA solution, washed sequentially with water and ethanol, and dried under a stream of nitrogen.

Assembly of Flow Cells [22]: The assays were performed in $75 \mu \mathrm{m}$ high and about $1 \mathrm{~cm}$ wide flow cells assembled from two coverslips and double-stick tape. In all flow cells, the bottom surface and top cover surface had identical surface chemistry. Solutions were exchanged within a few seconds by pipetting the new solution to one side of the cell and removing the old solution using filter paper from the other side.

Adsorption Process: A kinesin construct consisting of the wild-type, full-length Drosophila melanogaster kinesin heavy chain and a C-terminal His-tag was expressed in Escherichia coli and purified using a Ni-NTA column [46]. The density of functional motors in the eluent was estimated by this technique as described later. Microtubules were prepared by polymerizing $20 \mu \mathrm{g}$ of rhodamine labeled-tubulin (Cytoskeleton Inc., Denver CO) in $6.5 \mu \mathrm{L}$ of growth solution containing $4 \mathrm{mM} \mathrm{MgCl} 2,1 \mathrm{mM} \mathrm{GTP}$ and 5\% DMSO (v/v) in BRB80 buffer for $30 \mathrm{~min}$ at $37^{\circ} \mathrm{C}$ [47]. The microtubules with lengths between 1 and $20 \mu \mathrm{m}$ were 100 -fold diluted and stabilized in $10 \mu \mathrm{M}$ Taxol (Sigma, Saint Louis MO).

In one set of experiments (glass, PU and Pluronic surfaces), $0.5 \mathrm{mg} \mathrm{mL}^{-1}$ casein (technical grade, Sigma, Saint Louis, MO) dissolved in BRB80 buffer was adsorbed for $5 \mathrm{~min}$ to reduce denaturation of kinesin [21]. Next, diluted kinesin solution (kinesin stock solution, BRB80 buffer, $0.5 \mathrm{mg} \mathrm{mL}^{-1}$ casein, $1 \mathrm{mM}$ AMP-PNP from Sigma) was flowed in and after five minutes, exchanged against a $4 \mu \mathrm{g} / \mathrm{ml}$ microtubule solution (sheared thrice by passing through 30G1 needles from Becton Dickinson, Franklin Lakes, NJ) in BRB80 buffer containing $1 \mathrm{mM}$ AMP-PNP (Sigma-Aldrich, Saint Louis, MO), $0.2 \mathrm{mg} \mathrm{mL}^{-1}$ casein, $10 \mu \mathrm{M}$ Taxol, and an oxygen scavenging system to reduce photobleaching $(20 \mathrm{mM} \mathrm{D}$ - glucose, $0.02 \mathrm{mg} \mathrm{mL}^{-1}$ glucose oxidase, $0.008 \mathrm{mg} / \mathrm{mL}$ catalase, $10 \mathrm{mM}$ DTT) [48].

In another set of experiments (all surfaces except PU and Pluronic), casein adsorption was omitted and kinesin dilutions were made directly in BRB80 containing $1 \mathrm{mM}$ AMP-PNP but no casein. The microtubule solution was unchanged. Time elapsed since microtubule injection was measured using a digital stopwatch with one second accuracy.

Microscopy: An Eclipse TE2000-U fluorescence microscope (Nikon, Melville, NY) with a 40X oil objective (N.A. 1.30), an X-cite 120 lamp (EXFO, Ontario, Canada), a rhodamine filter cube (\#48002, Chroma Technologies, Rockingham, VT) and an iXon EMCCD camera (ANDOR, South Windsor, CT) and were used to image microtubules on the bottom surface of flow cells. Images were collected every 10 seconds with an exposure time of $0.5 \mathrm{~s}$.

Determination of landing rates and surface density: Microtubule landing events were manually counted within a field of view using UTHSCSA ImageTool version 3.0 and plotted against time elapsed after microtubule solution injection. The $N(\mathrm{t})$ plots were fitted (errorweighted least squares) with $N=N_{\max }\left[1-\exp \left(-R t / N_{\max }\right)\right]$ where $N$ is the number of landed microtubules (in a field of view of $0.04 \mathrm{~mm}^{2}$ ), $\mathrm{t}$ is the elapsed time and $\mathrm{R}$ is the initial landing rate specific to that motor density. However, for $(\mathrm{EG})_{3} \mathrm{OH}$ terminated SAM surfaces significant non-specific adsorption of microtubules was observed. This necessitated modification of the expression to
$N=N_{\text {init }}+N_{\max }\left[1-\exp \left(-R t / N_{\max }\right)\right]$ in order to account for the nonspecifically adsorbed MTs on the surface. The parameter $N_{\text {init }}$ is independent of time, since it was observed that in the absence of kinesin the number of non-specifically adsorbed microtubules does not change within the observation time beginning $100 \mathrm{~s}$ after microtubule injection. In the second PEGMA experiment, a small amount of nonspecific adsorption was observed, and the data points reflect a fit with $N_{\text {init }}, R, N_{\max }$ as parameters, while the error bars are extended to include the coverages derived from a fit with only $R$ and $N_{\max }$ as parameters.

The average microtubule length $L$ was measured by taking the arithmetic mean of at least 250 landed microtubules for every new microtubule preparation to calculate the area, $A=L w$ assuming a width $w$ of $25 \mathrm{~nm}$. The diffusion limited landing rate $Z$ is assumed to be equal to the landing rate observed on casein-coated glass surface at a very high kinesin solution concentration $(>10 \mathrm{nM}, 10$-fold dilution from stock solution), since a dilution series shows a saturation of landing rates reached for 40 -fold dilution of stock solution ( $\sim 5 \mathrm{nM}$ kinesin concentration) (Fig. 2g, h)). Using $A, Z$ (both measured on each day of experiments) and the landing rate $R$, the kinesin surface density can be calculated according to $\rho=-[\ln (1-R / Z)] / A$.

The dilution series data for casein-coated glass surfaces also enable an exact determination of the concentration of active kinesin motors in the stock solution under the experimentally validated assumption that all kinesin in the solution adsorbs uniformly to the casein-coated glass surface within 5 min [21]. The stock kinesin concentration is given by $C=2 \rho_{0} / h / N_{\mathrm{A}}$ where $h$ is the height of the flow cell and $\rho_{0}$ can be obtained by fitting the equation $R=Z\left[1-\exp \left(A \rho_{0} \xi\right)\right]$ to the landing rates on casein-coated glass surfaces as function of the dilution $\xi$ (Fig. 2h)

Received: August 8, 2007

[1] M. Q. Zhang, T. Desai, M. Ferrari, Biomaterials 1998, 19, 953.

[2] H. Ma, J. Hyun, P. Stiller, A. Chilkoti, Adv. Mater. 2004, 16, 338.

[3] L. Cao, M. Chang, C.-Y. Lee, D. G. Castner, S. Sukavaneshvar, B. D. Ratner, T. A. Horbett, J. Biomed. Mater. Res. A 2007, 81, 827.

[4] J. G. Archambault, J. L. Brash, Colloids Surf. B 2004, 33, 111.

[5] T. A. Horbett, Cardiovasc. Pathol. 1993, 2, S137.

[6] T. Fischer, H. Hess, J. Mater. Chem. 2007, 17, 943.

[7] Y. Hiratsuka, T. Tada, K. Oiwa, T. Kanayama, T. Q. Uyeda, Biophys. J. 2001, 81, 1555 .

[8] J. Clemmens, H. Hess, R. Lipscomb, Y. Hanein, K. F. Boehringer, C. M. Matzke, G. D. Bachand, B. C. Bunker, V. Vogel, Langmuir 2003, 19, 10967

[9] S. G. Moorjani, L. Jia, T. N. Jackson, W. O. Hancock, Nano Lett. 2003, 3, 633 .

[10] L. J. Cheng, M. T. Kao, E. Meyhofer, L. J. Guo, Small 2005, 1, 409.

[11] C. Reuther, L. Hajdo, R. Tucker, A. A. Kasprzak, S. Diez, Nano Lett. 2006, 6, 2177.

[12] M. G. van den Heuvel, C. T. Butcher, R. M. Smeets, S. Diez, C. Dekker, Nano Lett. 2005, 5, 1117.

[13] L. Ionov, M. Stamm, S. Diez, Nano Lett. 2005, 5, 1910.

[14] A. K. Boal, J. M. Bauer, S. B. Rivera, R. G. Manley, R. P. Manginell, G. D. Bachand, B. C. Bunker, Polym. Prepr. 2004, 45, 96.

[15] D. V. Nicolau, H. Suzuki, S. Mashiko, T. Taguchi, S. Yoshikawa, Biophys. J. 1999, 77, 1126.

[16] C. Mahanivong, J. P. Wright, M. Kekic, D. K. Pham, C. dos Remedios, D. V. Nicolau, Biomed. Microdev. 2002, 4, 111.

[17] M. Sundberg, M. Balaz, R. Bunk, J. P. Rosengren-Holmberg, L. Montelius, I. A. Nicholls, P. Omling, S. Tagerud, A. Mansson, Langmuir 2006, 22, 7302.

[18] G. S. Bachand, R. K.; Neves, H. P.; Olkhovets, A.; Craighead, H. G. Montemagno, C. D., Nano Lett. 2001, 1, 42.

[19] J. Yajima, M. C. Alonso, R. A. Cross, Y. Y. Toyoshima, Curr. Biol. 2002, 12, 301

[20] S. Diez, W. R. Schief, J. Howard, Curr. Biol. 2002, 12, R203. 
[21] J. Howard, A. J. Hudspeth, R. D. Vale, Nature 1989, 342, 154.

[22] J. Howard, A. J. Hunt, S. Baek, Methods Cell. Biol. 1993, 39, 137.

[23] C.-T. Lin, M.-T. Kao, K. Kurabayashi, E. Meyhöfer, Small 2006, 2, 281.

[24] J. Kerssemakers, J. Howard, H. Hess, S. Diez, Proc. Natl. Acad. Sci. USA 2006, 103, 15812.

[25] M. Schliwa, G. Woehlke, Nature 2003, 422, 759

[26] M. J. Wirth, D. J. Swinton, M. D. Ludes, J. Phys. Chem. B 2003, 107, 6258.

[27] S. M. Nie, R. N. Zare, Ann. Rev. Biophys. Biomol. Struct. 1997, 26, 567.

[28] R. D. Vale, B. J. Schnapp, T. S. Reese, M. P. Sheetz, Cell 1985, 40, 559.

[29] J. Howard, Mechanics of Motor Proteins and the Cytoskeleton, Sinauer, Sunderland, MA 2001

[30] M. J. deCastro, C. H. Ho, R. J. Stewart, Biochemistry 1999, 38, 5076.

[31] S. M. O'Connor, A. P. DeAnglis, S. H. Gehrke, G. S. Retzinger, Biotechnol. Appl. Biochem. 2000, 31, 185.

[32] J. Lahann, Polym. Int. 2006, 55, 1361.

[33] Y. Elkasabi, H. Y. Chen, J. Lahann, Adv. Mater. 2006, 18, 1521.

[34] K. Y. Suh, R. Langer, J. Lahann, Adv. Mater. 2004, 16, 1401.
[35] H. Y. Chen, J. Lahann, Anal. Chem. 2005, 77, 6909.

[36] J. Satulovsky, M. A. Carignano, I. Szleifer, Proc. Natl. Acad. Sci. USA 2000, 97, 9037.

[37] K. L. Prime, G. M. Whitesides, Science 1991, 252, 1164.

[38] S. Herrwerth, W. Eck, S. Reinhardt, M. Grunze, J. Am. Chem. Soc. 2003, 125, 9359 .

[39] E. Ostuni, R. G. Chapman, R. E. Holmlin, S. Takayama, G. M. Whitesides, Langmuir 2001, 17, 5605 .

[40] R. G. Chapman, E. Ostuni, L. Yan, G. M. Whitesides, Langmuir 2000, 16, 6927.

[41] C. Siegers, M. Biesalski, R. Haag, Chem. Eur. J. 2004, 10, 2831.

[42] M. Amiji, K. Park, Biomaterials 1992, 13, 682.

[43] D. Balazs, C. Hollenstein, H. Mathieu, Eur. Cells Mater. 2002, 3, 7

[44] D. Ji, C. M. Arnold, M. Graupe, E. Beadle, R. V. Dunn, M. N. Phan, R. J. Villazana, R. Benson, R. Colorado, T. R. Lee, J. M. Friedman, J. Cryst. Growth 2000, 218, 390.

[45] J. Lahann, R. Langer, Macromolecules 2002, 35, 4380.

[46] D. L. Coy, M. Wagenbach, J. Howard, J. Biol. Chem. 1999, 274, 3667.

[47] F. Gittes, B. Mickey, J. Nettleton, J. Howard, J. Cell Biol. 1993, 120, 923.

[48] A. Kishino, T. Yanagida, Nature 1988, 334, 74. 